\title{
Ride Comfort Improvements in a High-Speed Train with Active Secondary Suspension*
}

\author{
Anneli ORVNÄS**, Sebastian STICHEL** and Rickard PERSSON*** \\ **Department of Aeronautical and Vehicle Engineering, Royal Institute of Technology, \\ SE-100 44 Stockholm, Sweden \\ E-mail: orvnas@kth.se \\ ***Department of Vehicle Dynamics, Bombardier Transportation, \\ SE-721 73 Västerås, Sweden
}

\begin{abstract}
A combination of increased vehicle speeds and non-improved railway tracks may have a negative impact on passenger comfort. Active technology can replace the conventional passive solution of the secondary suspension of a rail vehicle in order to maintain good passenger comfort even when vehicle speed is increased and track conditions are inferior. This paper focuses on the benefits of using a so-called Hold-Off-Device (HOD) function in the lateral secondary suspension. On-track tests have been performed with the active secondary suspension concept implemented in a two-car Regina train during the summers of 2007 and 2008. The evaluated measurement results have been very satisfactory and the device will be implemented in long-term tests in service operation. These tests were carried out in the beginning of 2009.
\end{abstract}

Key words: Active Suspension, Hold-Off-Device, Ride Comfort, On-Track Tests

\section{Introduction}

Within the railway industry, there is an ambition to increase vehicle speed, in order to be able to compete with other means of transportation. However, increased train speed is, among other aspects, limited by track geometry, for example, if the lines are curvy. To build new dedicated straight lines for high-speed passenger trains is one possible solution. However, this is an extremely costly operation and it is not always an option in the short-term perspective. Another problem is track maintenance, which is also expensive. If maintenance is not prioritised, track conditions may deteriorate. With increased speed and inferior track conditions there is an imminent risk of negatively affected passenger comfort. One way to overcome this problem is to implement active technology in the secondary suspension of a rail vehicle. With active secondary suspension, passenger comfort can be maintained or even improved although vehicle speed is increased and track conditions are inferior.

Active secondary suspension in rail vehicles has been well studied for several decades. However, only a few service implementations have been made so far. The challenge of finding the right balance between good performance and acceptable costs remains, since implementing and maintaining active technologies may be expensive. However, modifications and improvements of the rail vehicle are generally less costly than modifications of the track. Since 2005, a Swedish research and development programme, Gröna Tåget (Green Train) ${ }^{(1)}$, is being carried out. Its main goal is to develop the next generation of high-speed trains for Nordic conditions. One part of the programme is to implement active secondary suspension in order to improve passenger comfort. So far,

[DOI: 10.1299/jmtl.3.206]

Copyright $(C 2010$ by JSME 
results have shown that the main advantages of using active lateral secondary suspension can be found in curves at high track plane acceleration ${ }^{(2)}$.

This paper describes the use of active technology in the lateral secondary suspension of a rail vehicle, focusing on the benefits of a Hold-Off-Device (HOD) function. On-track tests have been performed within the Gröna Tåget programme during the summers of 2007 and 2008. Measurement results from the tests in 2007 have been presented in Ref. (2). The corresponding results from 2008 are presented in this paper.

\section{Advantages of the HOD Function}

When travelling in a curve at high speed (high track plane acceleration), the carbody tends to move laterally outwards in relation to the track and bogies. The lateral carbody displacement is, however, limited by bumpstops. The gap between bogie frame and bumpstop (connected to the carbody) is $46 \mathrm{~mm}$ on the studied vehicle (Fig. 1). The bumpstop is a progressive rubber component with significant stiffness and after $65 \mathrm{~mm}$ there is a metallic stop. When bumpstop contact occurs it has a negative impact on ride comfort, since track irregularities are transmitted up to the carbody via the bumpstops without the normal filtering ability of the secondary suspension. Therefore, it is desirable to avoid bumpstop contact. If the lateral carbody displacement can be minimised, the risk of bumpstop contact is decreased and a smaller air gap between carbody and bogie can be allowed (bumpstop positions can be changed). In order to centre the carbody above the bogies in curves and thus to avoid bumpstop contact, a HOD function can be implemented.

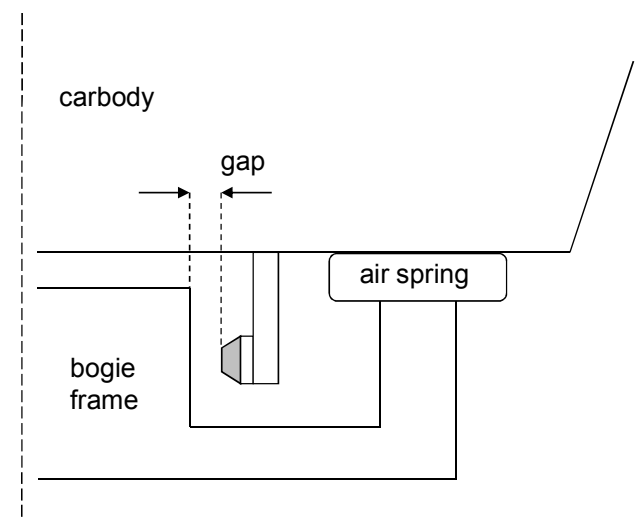

Fig. 1 Bumpstop connected to the carbody with a gap of $46 \mathrm{~mm}$ to the bogie frame

The HOD function is one part of the active lateral secondary suspension concept (ALS) and is based on low-pass filtered lateral bogie acceleration as the reference signal. Therefore, it is also called low-bandwidth control, since the reference signal is used for detecting the low-frequency content of deterministic track inputs, i.e. track geometry (curves). After low-pass filtering, the signal is multiplied with half the carbody mass in order to create an appropriate actuator force that counteracts the lateral movement of the carbody in the circular part of the curve. Hence, the carbody is centred above the bogies and bumpstop contact can be avoided. The HOD concept was introduced in the early 1990s by Allen ${ }^{(3)}$, when a prototype system using a hydraulic actuator was designed and tested.

The combination of the HOD function and bumpstops moved into new positions enables a wider carbody. The vehicle considered in the Gröna Tåget programme is a 3+2 seated Regina car, with an existing carbody width of $3450 \mathrm{~mm}$. By using the carbody centring HOD function, the profile is allowed to be increased by $100 \mathrm{~mm}$. The compliance with the vehicle gauge is ensured by the metallic stops, which are also moved to new positions. It should be noted that the benefit measured as increased carbody width is 
depending on which gauging standard that is applied. The increased width of the carbody may be used as larger interior passenger space, allowing wider seats, increased aisle width etc.

When a carbody is laterally displaced in relation to the track and bogies, so is the centre of mass of the carbody. This leads to a higher sensitivity to cross wind acting on the train and there is a risk of vehicle overturn. However, when the lateral movement of the carbody is restricted, due to changed bumpstop positions, the lateral displacement of the centre of gravity is also reduced. Hence, cross wind stability is increased and the risk of vehicle overturn is reduced.

In combination with the HOD function, the actuator has the ability to control the dynamic behaviour of the carbody. This is done by means of so-called sky-hook control, which is described thoroughly in Ref. (2). Dynamic control is most favourable on straight tracks, but it also positively affects ride comfort in curves.

\section{Description of the Actuator}

The hardware used for the tests within the Gröna Tåget programme is an electro-hydraulic actuator developed by Liebherr in Germany. It is a cylindrical damper with two chambers separated by a movable piston (Fig. 2). The chambers are provided with hydraulic fluid by means of two pumps, which are driven by an asynchronous motor fed by power from the train. A pressure difference between the two chambers is generated by controlling the outflow of the hydraulic fluid by means of pressure control valves. The pressure difference enables the actuator to create forces in both push and pull directions. In turn, the valves are controlled by varying voltage, determined by the force demand fed to the actuator. The actuator performs well in the frequency range up to $6 \mathrm{~Hz}$ and is able to generate a force response up to $30 \mathrm{kN}$.

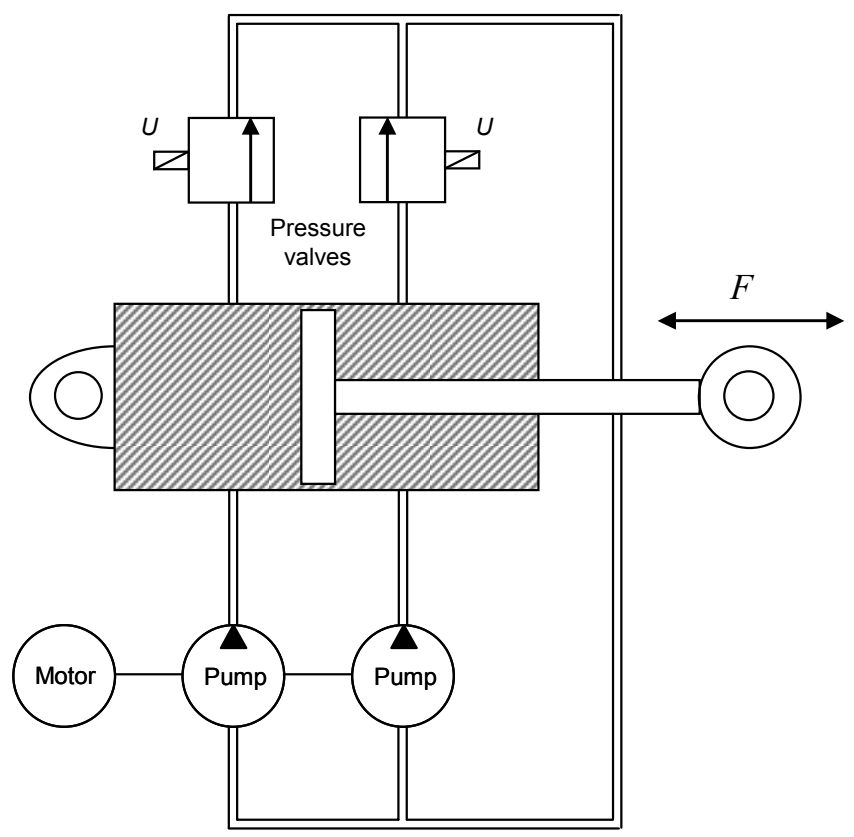

Fig. 2 Schematic picture of the electro-hydraulic actuator used in the present study

Apart from actuators, the active control system consists of sensors and a controller. The block diagram of the ALS can be seen in Fig. 3, where the signal from the bogie to the controller is an open-loop system, and the signal from the carbody to the controller forms a closed-loop system. 


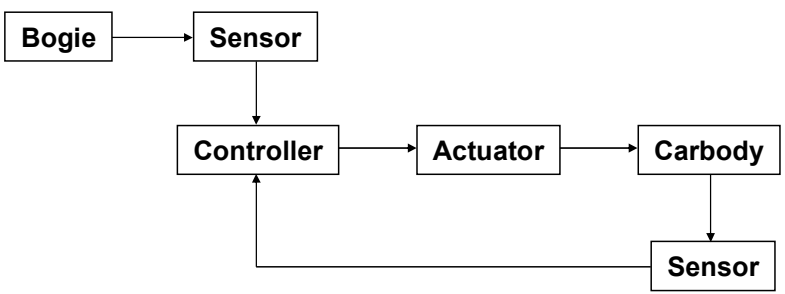

Fig. 3 Block diagram of the active lateral secondary suspension (ALS)

\section{On-Track Tests}

On-track tests within the Gröna Tåget programme have been performed during two summers (2007 and 2008). The tests have been performed in accordance with the vehicle certification requirements specified in UIC $518^{(4)}$. The same lines have been used on both occasions and the track conditions are considered to be the same, i.e. average to good for a $200 \mathrm{~km} / \mathrm{h}$ track in Sweden. Apart from tests on straight tracks, three different curve radius classes have been included: small-, medium- and large-radius curves, which correspond to 250-600 m (divided into $250-400$ and 400-600 m according to UIC 518), 900-1 $500 \mathrm{~m}$, and $3000-5000 \mathrm{~m}$, respectively.

The test vehicle is a two-car Regina train with ALS in one of the two cars. In the other car the passive damping was retained and could be used as reference when evaluating data from the car with active damping. Note that the actively damped car is called DMA and the passively damped car DMB, opposite to the notation from the tests in 2007 (see train configuration in Fig. 5).

The actuator used during tests in 2008 is generally the same as that in 2007 , i.e. an electro-hydraulic actuator (presented in the previous section). However, during the tests in 2007 there were some problems with overheating in very tough curving situations. Consequently, some modifications of the actuator were made before the tests in 2008, in order to reduce losses in the actuator and to increase heat radiation, and thus to avoid the overheating problem.

\subsection{Modifications from Previous Tests}

One modification of the suspension concept that was made in preparation for the tests in 2008 was that information from the leading bogie (its lateral acceleration) in the car with active damping was used for the actuator connected to the trailing bogie. The information was then used as curve detection by the HOD function in the trailing bogie. Hence, the actuator in the trailing bogie received more time-precise information and the delay could be minimised.

Furthermore, preparatory simulations before the tests in 2008 had shown that there is a difference in dynamic behaviour of the leading and trailing car of a vehicle. This difference is noticeable in the result evaluation both regarding lateral carbody acceleration and track forces. Therefore, it proved favourable with different dynamic damping parameters depending on the direction of travel, i.e. whether the actively damped car was leading or trailing. A parameter with information of the direction of travel was included in the control algorithm.

One of the challenges with active secondary suspension is to suppress lateral displacement when entering a curve, i.e. in transition curves. One way to minimise the lateral displacement in transition curves is to temporarily shut down the dynamic control in the transition curve; the dynamic control will otherwise keep the carbody in the tangent direction, increasing the displacement relative to the bogie. For this purpose a gyroscope has been used. The bogie roll velocity (roll rate) is zero on straight tracks and in the circular 
part of a curve. However, in transition curves, when the curve radius is continuously changing, there is a bogie roll velocity (Fig. 4). This information was given by the gyroscope and could be used in the control algorithm in order to detect transition curves and hence to control the dynamic damping.

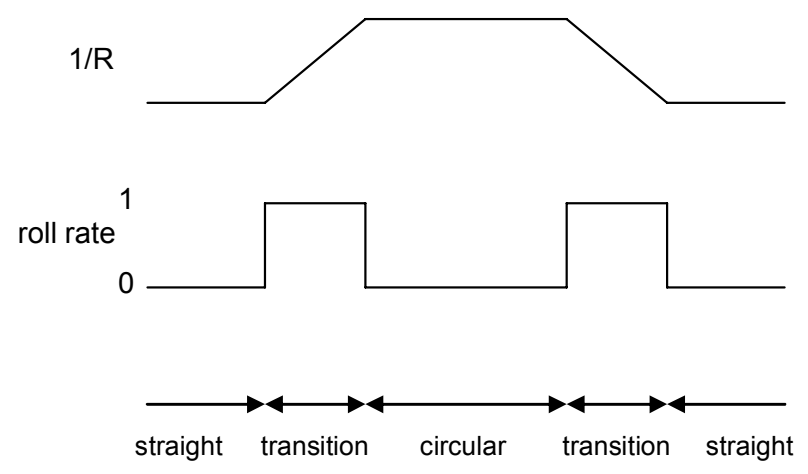

Fig. 4 Transition curve detection by means of a gyroscope

\section{Results}

\subsection{Evaluation of Measurement Results}

In order to assess the effect of active lateral secondary suspension on ride comfort, two different evaluation methods have been used, namely $\mathrm{Wz}$ and ISO. Wz is a frequency-weighted rms-value of the carbody acceleration over a one kilometre distance. It is most sensitive in the $4-7 \mathrm{~Hz}$ range. Ride comfort is considered to be comfortable for $\mathrm{Wz}$ between 2.0 and 2.5. Correspondingly, ISO is a frequency-weighted $r m s$-value of the carbody acceleration, evaluated over $5 \mathrm{~s}$. The weighting curve for horizontal comfort has its maximum in the $0.5-2 \mathrm{~Hz}$ range. Ride comfort is considered to be comfortable for ISO values between 0.2 and $0.3 \mathrm{~m} / \mathrm{s}^{2}$.

Due to the stiff coupling between the two carbodies, each car has an impact on the dynamic behaviour of the other (both positively and negatively). Furthermore, outer bogies, especially the last bogie, tend to behave differently compared to inner bogies. Therefore, it has been most correct to use a "mirror" comfort comparison between the DMA car (active) and the DMB car (passive); i.e. both end points of the carbodies are compared to each other (Fig. 5).

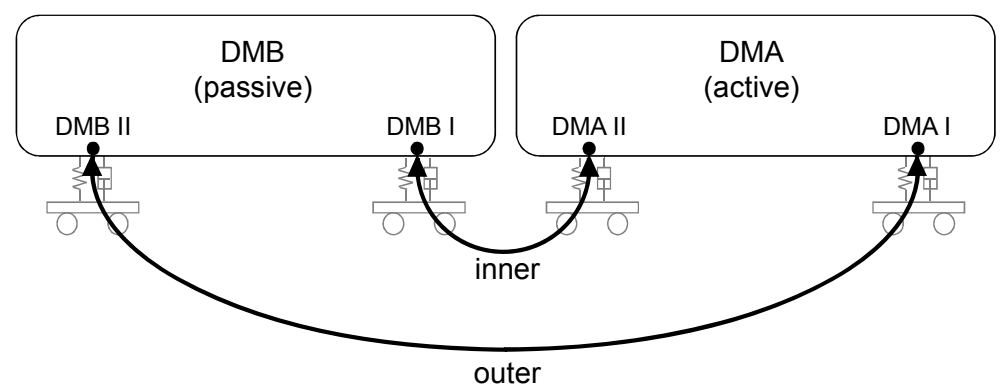

Fig. 5 "Mirror" comfort evaluation

Furthermore, the relative lateral displacement between carbody and bogies has been evaluated, since it indicates how well the HOD function is able to centre the carbody above the bogies. It shows how far the actively damped carbody is from bumpstop contact, and hence gives an indication on how narrow the gap between carbody and bogie can be. 


\subsection{The Comfort Values Wz and ISO}

Wz and ISO comfort evaluation from a number of tests performed during 2008 is shown in Fig. 6. The average comfort values over selected test sections of the outer ends of the carbodies are compared. In general, ride comfort is improved by active secondary suspension. However, it is obvious that comfort is dependent on the direction of travel.

Wz lateral

DMA leading, outer ends
Wz lateral

DMB leading, outer ends

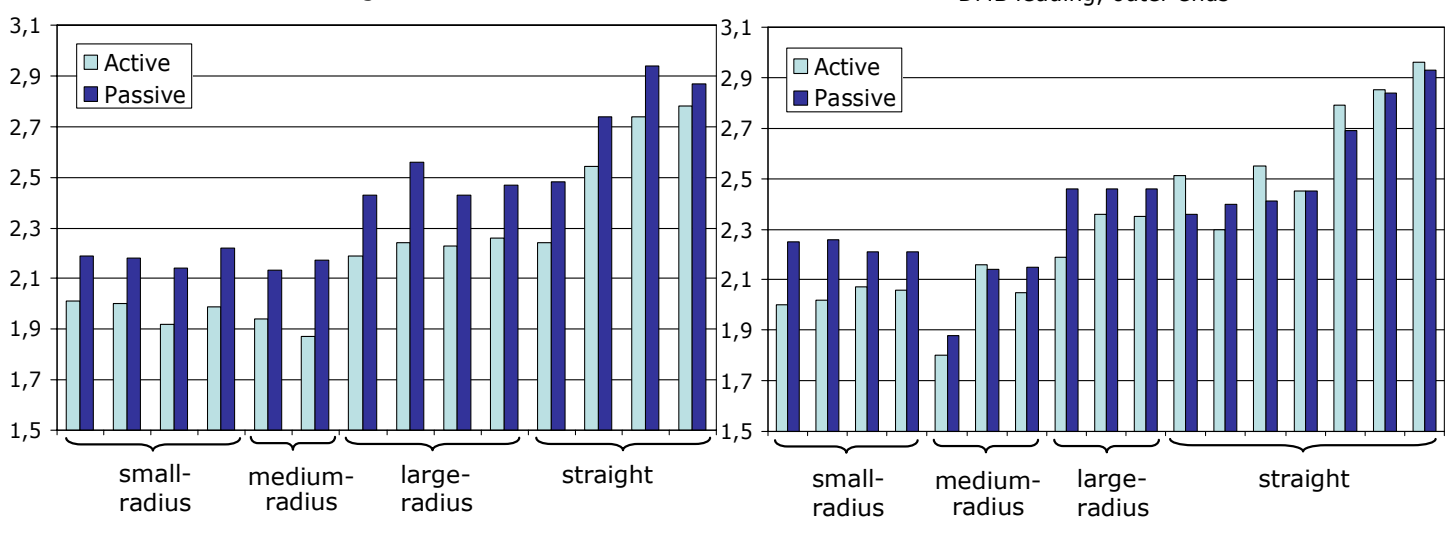

ISO lateral

DMA leading, outer ends

ISO lateral

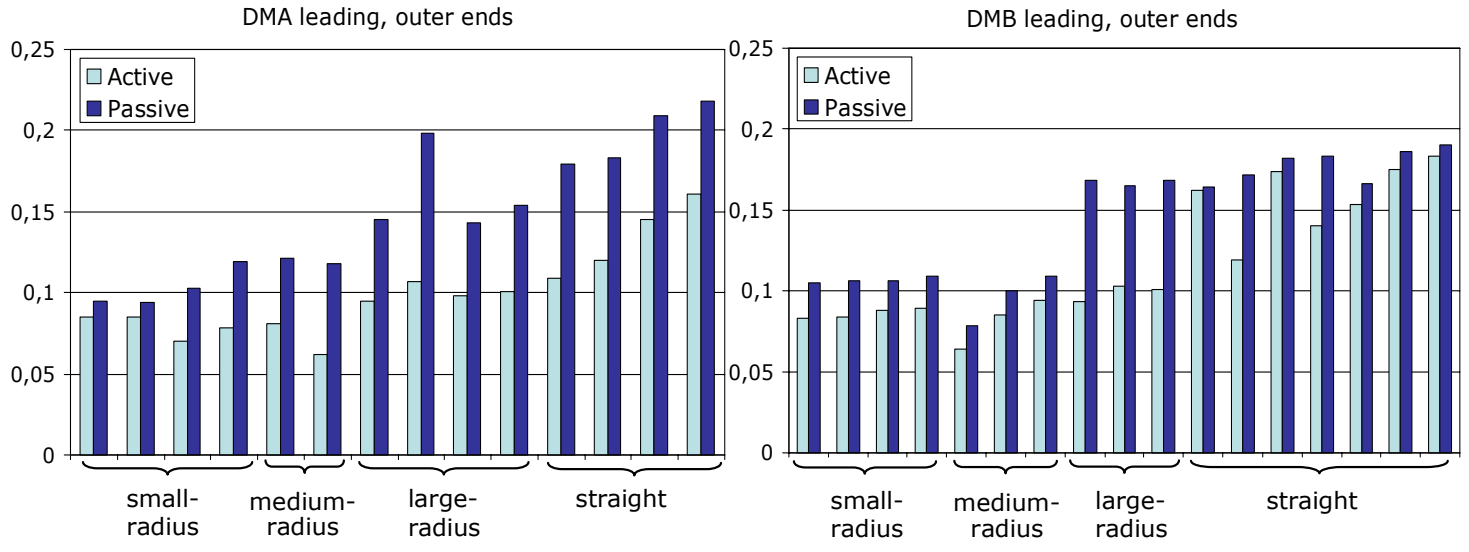

Fig. $6 \mathrm{Wz}$ and ISO evaluation of a number of tests, comparing outer ends of the carbodies

This effect can best be seen in the Wz values, where the leading car, on average, shows better comfort than the trailing car. The corresponding ISO values show a similar tendency, but the ride comfort of the car with active damping is, overall, better than in the car with passive damping. Furthermore, it can be seen that the ISO values generally indicate very good comfort for both the active and passive cases (comfortable between 0.2 and $0.3 \mathrm{~m} / \mathrm{s}^{2}$ ), whereas some $\mathrm{Wz}$ values exceed the limits for acceptable comfort (above 2.5). As explained above, $\mathrm{Wz}$ and ISO are evaluated according to different frequency ranges (different weighting filters). The reason why the ISO values show larger comfort improvements is that the control algorithm performs well up to approximately $3 \mathrm{~Hz}$, which overlaps the sensitivity range of the ISO evaluation $(0.5-2 \mathrm{~Hz})$.

The largest difference in $\mathrm{Wz}$ values between the actively and the passively damped carbodies is 0.24 units in small-radius, 0.30 in medium-radius, 0.32 in large-radius curves and 0.24 on straight track. The corresponding figures for the ISO values show an improvement of $34 \%$ in small-radius, $47 \%$ in medium-radius, $46 \%$ in large-radius curves and $39 \%$ on straight track. 


\subsection{Relative Lateral Displacement between Carbody and Bogies}

The relative lateral quasi-static displacements between carbodies and bogies have been evaluated for all tests performed during 2008 in curves with radii from 300 to $3200 \mathrm{~m}$. A summary of all tests with the passively damped DMB car as leading can be found in Fig. 7 . It can be seen that the active secondary suspension (HOD function) in the actively damped DMA car manages to minimise the lateral displacement of the carbody in relation to the bogies. Although the bumpstops are already moved to new positions in the DMA car (26 mm instead of $46 \mathrm{~mm}$, as in the DMB car), bumpstop contact is avoided. For track plane accelerations up to around $1.1 \mathrm{~m} / \mathrm{s}^{2}$ the lateral carbody displacement in relation to the bogies is well suppressed. For higher track plane accelerations the actuator force response is limited and the relative displacement increases; however, bumpstop contact is still avoided. The corresponding evaluation of the passively damped DMB car shows that the relative lateral displacements between carbody and bogies cause bumpstop contact at track plane acceleration as low as around $0.9 \mathrm{~m} / \mathrm{s}^{2}$; hence, ride comfort is significantly reduced.

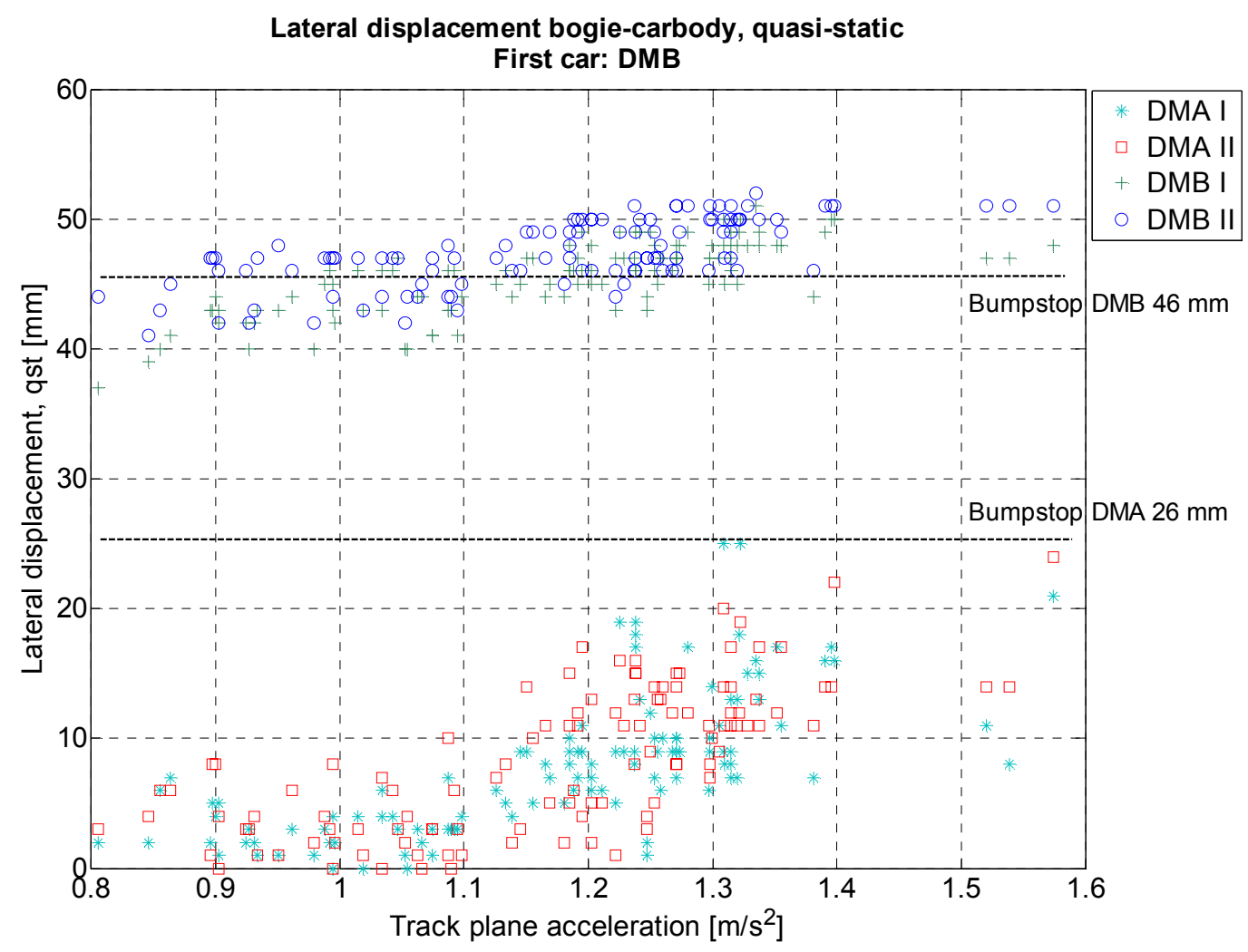

Fig. 7 Relative lateral quasi-static displacements between carbodies and bogies as a function of track plane acceleration (DMA active, DMB passive)

Also Fig. 8 illustrates the effects of the HOD function, showing measurement results of the relative lateral displacements between carbodies and bogies from one test performed in 2007 and the corresponding test in 2008. The test section is located between Ange and Sundsvall with curve radii 270-300 $\mathrm{m}$ (small-radius curves) and corresponding track plane accelerations $1.0-1.2 \mathrm{~m} / \mathrm{s}^{2}$. Both tests were performed with the passively damped carbody leading. The upper two diagrams show the relative displacements of the leading passively damped carbody and its respective bogies. No modifications of the passively damped carbody were made between the two test periods and the measurement results should be and are similar. The passively damped carbody moves laterally outwards in the curves in relation to the bogies and bumpstop contact occurs at the relative displacement of $46 \mathrm{~mm}$. 
The lower two diagrams show the relative displacements of the trailing actively damped carbody and its respective bogies. The diagrams clearly show that the HOD function manages to centre the actively damped carbody above the corresponding bogies. Moreover, an improvement of the vehicle dynamic behaviour between the two test periods in 2007 and 2008 can be noticed. It can be seen that the centring HOD function generally works better for the test in 2008 compared to that in 2007. The relative displacement of the 2007 test of the trailing active bogie (lowest diagram) is somewhat higher in the curve sections than that of the leading active bogie (second lowest diagram). In 2008 the relative displacement of the trailing active bogie was suppressed in transition curves due to the information used from the leading active bogie (its lateral acceleration), which has been explained in $\$ 4.1$.
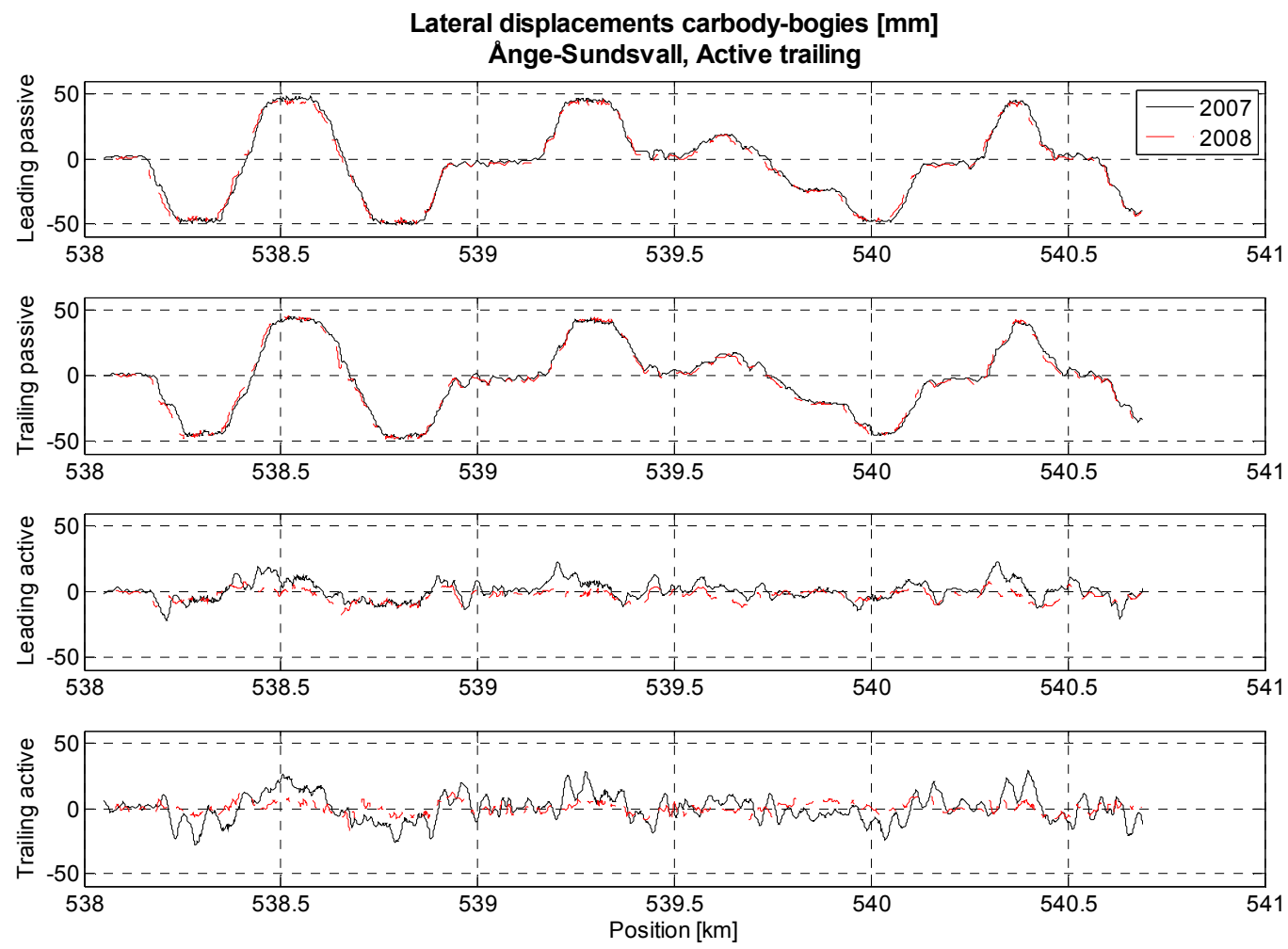

Fig. 8 Relative lateral displacements between carbodies and bogies from tests during 2007 and 2008, respectively. Ånge-Sundsvall, curve radii $270-300 \mathrm{~m}$, speed $85-87 \mathrm{~km} / \mathrm{h}$, track plane acceleration $1.0-1.2 \mathrm{~m} / \mathrm{s}^{2}$

One modification that was made to the tests in 2008 is the use of a gyroscope in order to detect transition curves (mentioned in $\$ 4.1$ ). The effect of this feature can be seen in Fig. 9. The relative lateral displacements of the car with active damping from two tests (51 and 55) are plotted as a function of position along the track (between Uppsala and Sala). The lowest diagram illustrates the curvature of the track, where transition sections and circular parts of the curves can be identified. Test 51 was performed with full damping control in transition curves. In test 55, the gyroscope was used in order to detect transition curves so that the dynamic damping in these sections could be switched off. It can be seen that the relative lateral displacements between carbody and bogies are lower in transition curves when the active damping is not in use (test 55). 

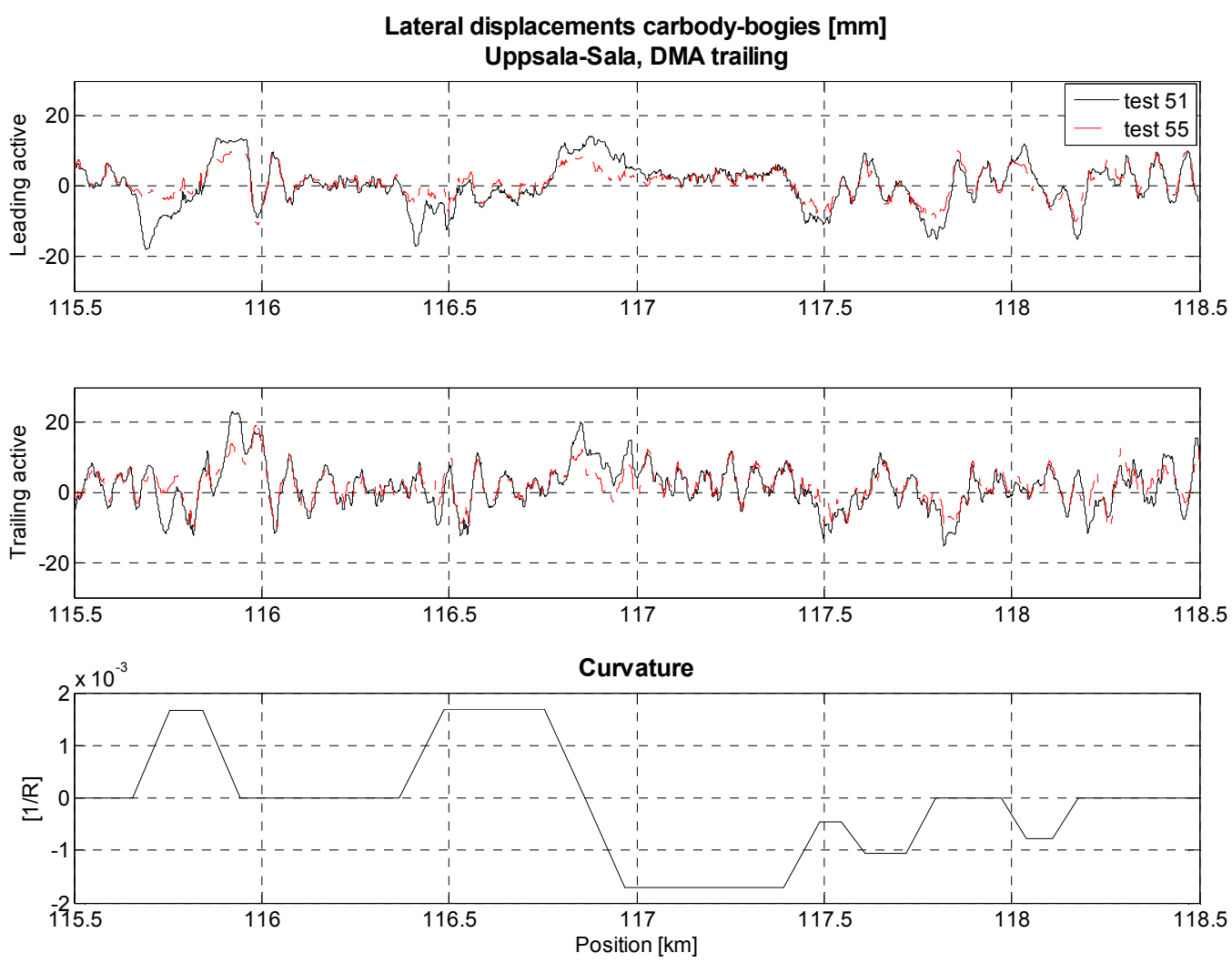

Fig. 9 Relative lateral displacements between carbody and bogies (DMA active) and the track curvature. Uppsala-Sala, curve radius $600 \mathrm{~m}$, speed $122 \mathrm{~km} / \mathrm{h}$, track plane acceleration $0.93 \mathrm{~m} / \mathrm{s}^{2}$

\section{Conclusions}

Active secondary suspension in a rail vehicle has been evaluated through on-track tests performed on selected tracks in Sweden during the summers of 2007 and 2008. Especially the carbody centring HOD function of the active suspension has shown to significantly improve ride comfort, since bumpstop contact can be avoided. Evaluation of measurement results from the tests in 2008 have shown improvements of the vehicle dynamic behaviour compared to the tests performed in 2007.

A comparison of comfort values of the actively and passively damped carbodies from the tests in 2008 show that the active suspension improves Wz by up to 0.24 units and ISO by $34 \%$ in small-radius curves, up to $0.30 \mathrm{Wz}$ units and $47 \%$ ISO in medium-radius curves, up to $0.32 \mathrm{Wz}$ units and $46 \%$ ISO in large-radius curves, and up to $0.24 \mathrm{Wz}$ units and $39 \%$ ISO on straight track.

The test train with active secondary suspension fulfils the UIC 518 requirements for a maximum speed of $250 \mathrm{~km} / \mathrm{h}$ and a maximum cant deficiency of $183 \mathrm{~mm}$. The satisfactory results enable implementation of active secondary suspension in long-term tests in service operation. These tests were carried out in the beginning of 2009.

\section{Acknowledgements}

The research and development programme, Gröna Tåget (Green Train), within which the present study has been performed, is financed by Banverket (Swedish Rail Administration). 


\section{References}

(1) Homepage of Gröna Tåget (Green Train), www.gronataget.se.

(2) Orvnäs, A., Stichel, S. and Persson, R., On-Track Tests with Active Lateral Secondary Suspension: A Measure to Improve Ride Comfort, ZEVrail Glasers Annalen, Vol. 132, No. 11-12 (2008), pp. 469-477.

(3) Allen, D. H., Active Bumpstop Hold-Off Device, Proc IMechE Conference Railtech 94, paper C478/5/013, 1994.

(4) UIC Code 518, Testing and Approval of Railway Vehicles from the Point of View of Their Dynamic Behaviour - Safety - Track Fatigue - Ride Quality, third edition, October 2005. 